\title{
Neoplastic Lesions of Small and Large Intestine- A Prospective Study in Tertiary Care Hospital
}

\author{
Venkata Kalyan Nunna, Prabhu M H*, S S. Inamdar. Mallikarjun Pattanashetti and Rachana L. Y \\ Department of Pathology, S. N.Medical College, Bagalkot, Karnataka,India
}

\begin{abstract}
Background:This study was carried out to study the occurrence, evaluate and correlate various histopathologic types of tumors at different sites of gastrointestinal tract in relation to age and sex of the patients operated in Surgery department and specimens received at Pathology department of S.Nijalingappa Medical college, Bagalkot,Karnataka.

Methods: A cross sectional histopathological study of gastrointestinal tumors of 45 cases was carried out on surgical specimens and biopsy received from Department of Surgery, S.Nijalingappa Medical College, Bagalkot from January 2016 to July 2017. Specimen were preserved in $10 \%$ buffered formalin. It was kept in $10 \%$ buffered formalin for proper fixation, subsequently dehydration, clearing, embedding in paraffin wax was carried out. Blocks were made and sections of $3 \mu \mathrm{m}$ thickness were cut and stained with Haematoxylin and Eosin stain and slides were reported.

Results: A total of 45 cases were included, among which 26 were males and 19 were females with peak occurrence in sixth decade.The following is anatomical distribution : Small intestine 7(15.5\%), Appendix 0(0\%), Colon \& Rectum $37(82.2 \%)$ and Anal canal 01(2.2\%). Histological types seen were - Epithelial 41(91.1\%), Mesenchymal 03 (6.6\%) and Lymphoma 01(2.2\%).

Conclusion: Gastrointestinal tumors show a wide variation in the morphology.Hence, histopathological examination is must for the diagnosis and classification of these tumors. The occurrence of gastrointestinal tumors was mostly in the colon and rectum. This study emphasises the need for early diagnosis of the disease through histopathology, which when correlated clinically will help the clinician to implement the appropriate treatment and improve the survival of the patients.
\end{abstract}

Keywords: Histopathological Study, Gastrointestinal Tumors, Biopsy.

\section{Introduction}

The small intestine and large intestine accounts for most of the gastrointestinal tract length and are the sites of a broad array of diseases. Gastrointestinal tumors account for a large proportion of all neoplasms. ${ }^{[1]}$ Colorectal cancer ranks second among the most common tumors of the world, according to the World Cancer Report of 2000.

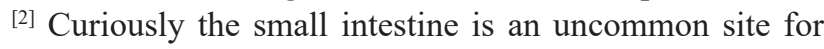
tumor despite its great length and vast pool of dividing cells. ${ }^{[1]}$ There is world wide variation in the distribution of these neoplasms, which appear largely due to exogenous factors rather than genetic factors. ${ }^{[3]}$ The various histologic types of tumor at different intestinal sites also differ in their incidence and prognosis. Tumors arising from the mucosa of intestines predominate over mesenchymal and stromal tumors. Adenocarcinomas constitute $70 \%$ of all malignancies arising in the intestinal tract. All tumors without exception are incurable when metastasis exists. However effective treatment in case of lymphoma and stromal tumors is likely to result in cure. ${ }^{[1]}$ This study is undertaken to determine the relative frequency of various histopathologic types of lesions of intestines in this part of the country. Knowledge about their prognosis will help the clinician in effective management of patient.

\section{Materials and Methods}

This is a cross sectional study done in the Department of Pathology, S.Nijalingappa Medical College, Bagalkot from January 2016 to July 2017. Histopathological study of 45 cases of gastrointestinal tumors was carried out on surgical specimens and biopsy received from Department of Surgery after taking approval from Institutional Ethical Committee of our Institution. Written and informed consent was taken from all the patients after a brief explanation of the procedure. A careful clinical history including demographic data like age was taken. Specimens received were sent in $10 \%$ buffered formalin. The biopsy and surgical specimens obtained was fixed in $10 \%$ buffered formalin. Formalin fixed specimens were subjected to detailed gross examination and subjected for histopathological processing and paraffin blocks prepared. Sections were cut at 3-5 $\mu \mathrm{m}$ thickness and stained by Hematoxylin and Eosin and mounted with DPX. The slides thus prepared were then examined under the microscope, and the lesions were diagnosed and classified according to the recent WHO 
classification. The data obtained was coded and entered into Microsoft Excel Spreadsheet. The data was analyzed using SPSS version 20. Categorical data was expressed in terms of rates, ratios and percentage.

\section{Results}

A total of 45 cases: 26 males and 19 females were included. In our study, gastrointestinal tumors were seen over the age range of 6 years to 80 years. The maximum number of tumours were seen in the $6^{\text {th }}$ decade. Male predominance was observed in this study with a male to female ratio of 1.36:1 The following is anatomical distribution : Small intestine 7(15.5\%), Appendix 0(0\%), Colon \& Rectum 37 $(82.2 \%)$ and Anal canal 01(2.2\%).Histological types seen were - Epithelial 41(91.1\%), Mesenchymal 03 (6.6\%) and Lymphoma 01(2.2\%) as depicted in Table 1. The following histological types were reported - epithelial 41 (91.1\%), mesenchymal 03 (6.6\%) and Lymphoma 01(2.2\%) as depicted in Table 2. Majority of the cases were Moderately differentiated adenocarcinomas $(65.7 \%)$ as tabulated in
Table 3. Neoplastic lesions in small intestine and large intestine accounted for $15.5 \%$ and $82.2 \%$ respectively in our study. The anatomical site commonly involved was Recto-Sigmoid region, constituting $86.4 \%$ of all colorectal malignancies.

Mucinous adenocarcinoma of colon, Signet ring cell carcinoma, Gastrointestinal Stromal Tumor of Jejunum - Spindle cell type and Malignant lymphoma - Diffuse Large B Cell Lymphoma of Ileum are shown in Figure 1, Figure 2, Figure 3 and Figure 4 respectively. Table 4 depicts the histological variants of Colorectal carcinoma which includes Adenocarcinoma (80.5\%), Mucinous Adenocarcinoma (11.1\%), Signet ring cell carcinoma $(5.5 \%) \&$ Squamous cell carcinoma $(2.7 \%)$. There were a total 7 cases of small intestinal tumors with following location : Duodenum 01(14.2\%), Jejunum 02(28.5\%) and Ileum $04(57.1 \%)$. The distribution of 37 cases of colorectal tumors are Caecum 02(5.4\%), Ascending colon 03(8.1\%) and Rectosigmoid colon 32(86.4\%) cases.

Table 1: Anatomical distribution of Gastrointestinal Tumors.

\begin{tabular}{|l|c|c|}
\hline Site & Number & Percentage \\
\hline Small intestine & 07 & $15.5 \%$ \\
\hline Large intestine & 37 & $82.2 \%$ \\
\hline Anal canal & 01 & $2.2 \%$ \\
\hline
\end{tabular}

Table 2: Histological types of Gastrointestinal Tumors.

\begin{tabular}{|l|c|c|c|c|c|}
\hline Site & Small intestine & Large intestine & Anal canal & Total & percentage \\
\hline Epithelial & 04 & 36 & 01 & 41 & 03 \\
\hline Mesenchymal & 02 & 01 & - & $01.1 \%$ & $6.6 \%$ \\
\hline Lymphoma & 01 & - & - & $\mathbf{4 5}$ & $2.2 \%$ \\
\hline Total & & & & $\mathbf{1 0 0}$ \\
\hline
\end{tabular}

Table 3: Differentiation of Gastrointestinal Tumors

\begin{tabular}{|l|c|c|c|}
\hline Differentiation & Small intestine & Large intestine & Anal canal \\
\hline Well & $01(25 \%)$ & $05(13.8 \%)$ & $01(100 \%)$ \\
\hline Moderately & $02(50 \%)$ & $28(77.7 \%)$ & - \\
\hline Poorly & $01(25 \%)$ & $03(8.3 \%)$ & - \\
\hline Total & $\mathbf{0 4}$ & $\mathbf{3 6}$ & $\mathbf{0 1}$ \\
\hline
\end{tabular}

Table 4: Histological Variants of Colorectal Carcinoma.

\begin{tabular}{|l|c|c|}
\hline Variants & Number & Percentage \\
\hline Adenocarcinoma & 29 & 80.5 \\
\hline Mucinous adenocarcinoma & 04 & 11.1 \\
\hline Signet ring cell carcinoma & 02 & 5.5 \\
\hline Squamous cell carcinoma & 01 & 2.7 \\
\hline Total & 36 & 100 \\
\hline
\end{tabular}




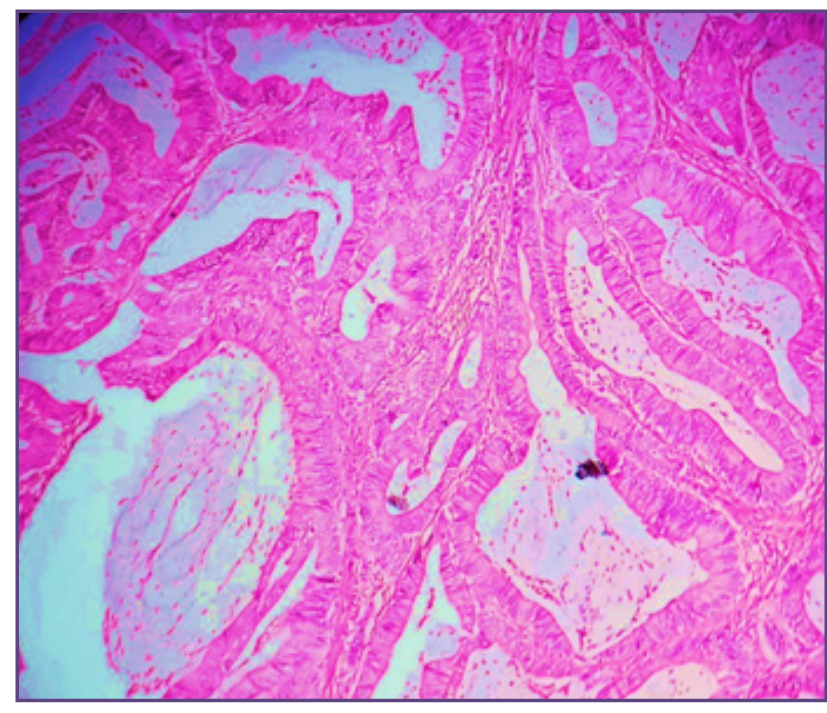

Fig. 1: Photomicrograph of Mucin secreting adenocarcinoma of caecum- large intestine (H \& E Stain, 100X).

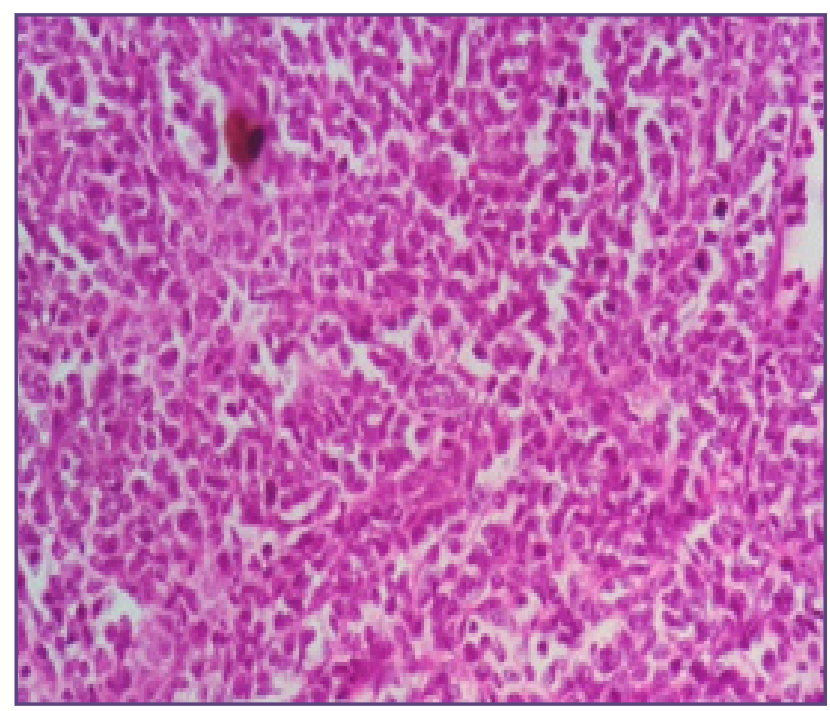

Fig. 3: Photomicrograph of Diffuse Large B Cell Lymphoma of Ileum (H \& E Stain, 400X).

\section{Discussion}

WHO Histological Classification of tumors of the small intestine includes Epithelial tumors, Non-Epithelial tumors , Malignant lymphomas, Secondary tumors and Polyps. Epithelial tumors are classified as Adenomas, Intraepithelial neoplasia (dysplasia) associated with chronic inflammatory diseases and Caricnomas. Non epithelial tumors include Lipoma, Leiomyoma, GIST, Leiomyosarcoma, Angiosarcoma and Kaposi sarcoma. Malignant lymphomas includes Immunoproliferative Small intestinal disease, Western type B-cell lymphoma of MALT, Mantle cell lymphoma, DLBCL Burkitt

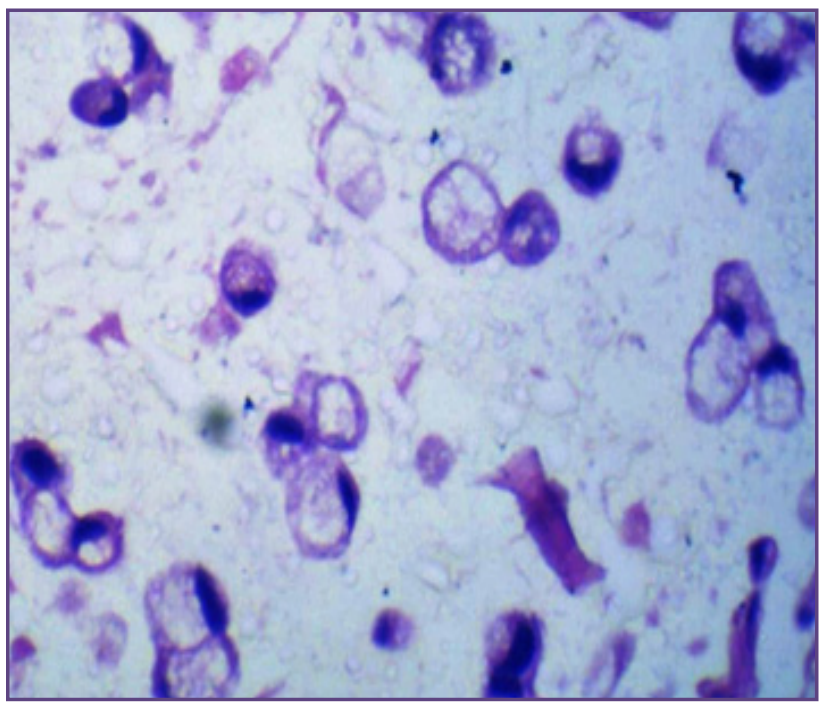

Fig. 2: Photomicrograph of Signet ring adenocarcinoma of rectum (H \& E Stain, 400X).

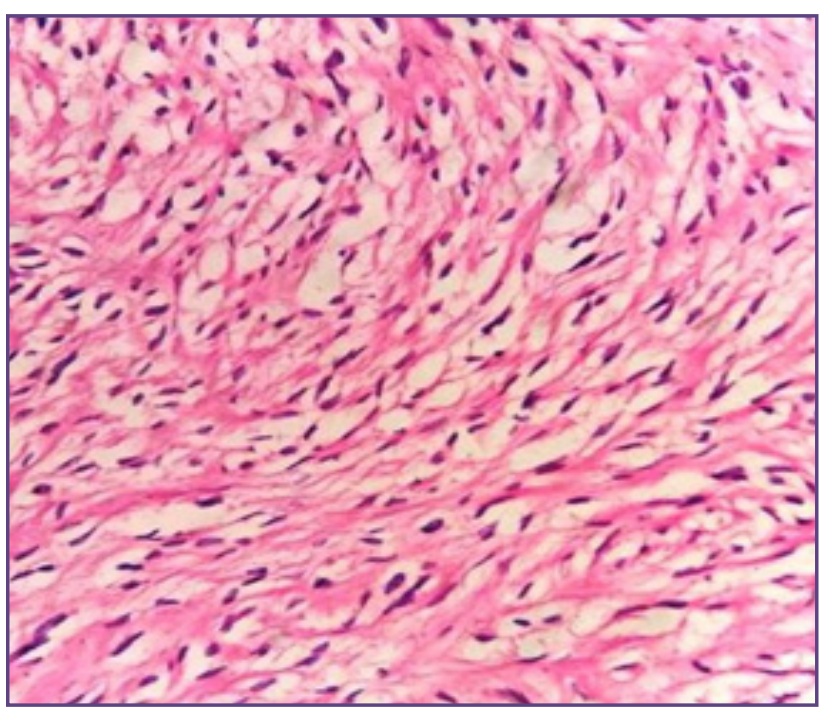

Fig. 4: Photomicrograph of Gastrointestinal Stromal tumour of jejunum (H \& E Stain, 400X).

Lymphoma, Burkitt-like/Atypical Burkitt-lymphoma,Tcell lymphoma and other rare lymphomas. The polyps are classified as Hyperplastic, Peutz Jeghers and juvenile polyps. ${ }^{[4]}$

GISTs are mesenchymal neoplasms of the gastrointestinal tract that express C-kit, with rare exceptions. Two thirds arise from stomach and one fourth from small intestine and the rest occurs in large intestine. The macroscopic features show usually solitary, rounded or ovoid mass varying in size from $2-20 \mathrm{~cm}$. On cross section they are circumscribed, lack a true capsule and reveal a pink or grey cut surface with rubbery consistency. They may have 
areas of haemorrhage, necrosis, myxoid change or cavitary degeneration. ${ }^{[5]}$ They are categorized into Spindle cell type (70\%),Epithelioid type (20\%) and Mixed type.

Adenocarcinomas constitute $>95 \%$ of colorectal cancers. Colorectal carcinoma is the third most common cancer in men and the second in women worldwide. Cancer of the colon is not a very common disease in our country and the incidence is much lower than in the western world. The incidence in India is about 7/100,000. ${ }^{[6]}$ A major etiological factor is lifestyle involving diet rich in fat, refined carbohydrates and animal protein, combined with low physical activity. Sequential genetic alterations mediate development of colon cancer, the earliest such change being mutation of the APC gene. A subset of cancer with sporadic microsatellite instability is associated with female gender, proximal location and younger age. Precancerous conditions include adenomas, FAP, chronic inflammatory bowel disease, juvenile polyposis and following pelvic irradiation. ${ }^{[5]}$

Gastrointestinal tumors were seen over a wide range of age (6 years to 80 years). The highest distribution was found in the $6^{\text {th }}$ decade, which was consistent with the study by Assem O. et al[ ${ }^{[7]}$ and Patel M M et al ${ }^{[8]}$ However the peak distribution was seen at $7^{\text {th }}$ decade in study done by Mohammad et al. ${ }^{[3]} \mathrm{A}$ male predominance was observed in this study with a male to female ratio of $1.36: 1$ which is consistent with study by Shahid Jamal et al, 2005 ${ }^{[9]}$, while male to female ratio is $1.67: 1$ and $1.39: 1$ in studies by Assem et al and Patel et al ${ }^{[10]}$ respectively. The histological types of gastrointestinal tumors show immense variation. However, adenocarcinomas were the commonest tumors all over the gastrointestinal tract. The present study also showed similar findings. The anatomical site commonly involved was Recto-Sigmoid region, constituting $86.4 \%$ of all colorectal cancers which was consistent with study by Leena et al. In our study, the neoplastic lesions in small intestine and large intestine accounted for $15.5 \%$ and $82.2 \%$ respectively whereas study conducted by Nannavati MG et al ${ }^{[1]}$ found a slightly lower incidence of $9.3 \%$ in small intestine and slightly higher incidence of $86 \%$ in large intestine.

\section{Conclusion}

Gastrointestinal tumors show a wide variation in the morphology. Histopathological examination is must for the diagnosis and typing of these tumors. It can be concluded from the present study that the malignant lesions are more common in colorectum. Any adult with complaints of vague abdominal pain, blood or mucus in the stool or features of hemorrhoids which may herald the onset of colorectal carcinoma should be adequately investigated. Our study emphasises the need for early diagnosis of the disease by histopathology, which when correlated clinically will help the treating doctor to implement the appropriate treatment and hence improve the survival of the patients.

\section{Acknowledgement}

The authors would like to thank the patients for the support. We also thank the staff of Department of Pathology and Department of Surgery, S Nijalingappa Medical College, Bagalkot Karnataka for their assistance.

\section{References}

1. Turner J R. The Gastrointestinal Tract. In: Vinay Kumar, Abul Abbas and Jon Aster. Robbins and Cotran Pathologic Basis of Disease; 9th Edition. Elsevier Health Sciences. London, 2014;777-819.

2. Norio Matsukura, Hiroko Ohgaki, Rens Lambert. World Cancer Report,Lyon,France, IARC Press. 2003:194-202.

3. Mohammad A, Makaju R. Retrospective histopathological analysis of various neoplasms of different parts of the gastrointestinal tract seen at the Kathmandu University Teaching Hospital. Nepal. Kathamandu University Medical Journal 2005;4:474-8.

4. Bosman F.T, Carnerio F, Hruban R.H, Theise N.D. Pathology and Genetics. Tumors of Digestive system. WHO classification of tumors, 4th Edition, Lyon, France: IARC Press International Agency for Research on Cancer 2010:210-31.

5. Hornick L.J. Tumor of the small and large intestines, including anal region. In: Christopher Fletcher. Fletcher's Histopathology of Tumors. 4th Edition. Volume 1. Churchill Livingstone. Elsevier Health Sciences. London 2013; 434 76.

6. Sharma P, Deka M. A Study of Neoplastic Lesions of Colorectum in a Tertiary Care Hospital. Int J Sci Stud. 2015;3(8):88-91.

7. Assem O. et al. Primary gastrointestinal cancers in the Western Region of Saud Arabia. Saudi Medical Journal. 2000;21(8):730-4.

8. Patel MM, Gamit B, Patel PR. Analysis of gastrointestinal malignancy: A Five Years study. National Journal of Community Medicine. 2012;3(3):555-7.

9. Shahid J, Nadira M, Sajid M, Muhammad L. Analysis of Gastrointestinal malignancy at Armed Forces Institute of Pathology, Ravalpindi, Pakistan. Asian pacific journal of cancer prevention 2005;6:497-500. 
10. KK Leena Devi, N Suvarna. Patterns of Gastrointestinal tumors in North Kerala. Indian Journal of Cancer 1980;17:159-63.
11. Nannavati MG, Parikh JH, Gamit KS, Modh SD. A Histopathological Study of Intestinal Lesions. International Journal of Science and Research 2014;3(9):326-30 .

*Corresponding author:

Dr Prabhu M H, Associate Professor, Department of Pathology, S Nijalingappa Medical College, Bagalkot Karnataka

Phone: +91 9243529531

Email: docprabhumh@gmail.com

Financial or other Competing Interests: None. 